Original Research Paper

\title{
Black Tea Extract on the Preservation of Cantonese Sausage
}

\author{
${ }^{1}$ Huan Cheng, ${ }^{2}$ Ying Liu, ${ }^{1}$ Dan Wu, ${ }^{1}$ Xiaona Zhao, ${ }^{1}$ Chenlan Mao, ${ }^{1}$ Shuping Wang and ${ }^{1}$ Jianchu Chen \\ ${ }^{1}$ College of Biosystems and Food Science, Zhejiang University, Hangzhou, China \\ ${ }^{2}$ Department of Food Nutrition and Detection, Hangzhou Vocational and Technical College, Hangzhou, China
}

\author{
Article history \\ Received: 17-06-2020 \\ Revised: 13-08-2020 \\ Accepted: 02-09-2020 \\ Corresponding Author: \\ Dan Wu \\ College of Biosystems and \\ Food Science, Zhejiang \\ University, Hangzhou, China \\ Email:wudan2008@zju.edu.cn
}

\begin{abstract}
Black Tea Extract (BTE) is a natural pigment with strong antioxidant activity. Its effect on the preservation of Cantonese sausage was investigated based on polyphenol components analysis in this study. The content of tea polyphenols in BTE was $26.61 \%$ by using spectrophotometry, the tea catechin in BTE was analyzed by HPLC, which included gallic acid $(0.27 \%)$, caffeine $(5.75 \%)$ and 8 catechins $(0.87 \%$ Catechin, $1.66 \%$ epicatechin, $0.34 \%$ catechin gallate, $2.54 \%$ epicatechin gallate, $1.88 \%$ gallocatechin, $5.31 \%$ epigallocatechin, $2.69 \%$ gallocatechin gallate, $10.23 \%$ epigallocatechin gallate). The tea sausages of five treatments with the addition of $0.05,0.10,0.15,0.20$ and $0.25 \%$ BTE were designed, compared to the control and the sausage with the addition of $0.02 \%$ sodium Nitrite (NIT) and the addition of $0.01 \%$ NIT and $0.01 \%$ BTE. The acid value, peroxide value and sensory indices were analyzed and the shelf-life of the sausage was predicted by peroxide value. The sausage with $0.15 \%$ BTE had significant improved the product quality by reducing the acid value and lipid oxidation value. The nitrite and BTE had synergistic inhibition of acid value increase. The BTE had the positive effect on the color of the freshly prepared sausage, but this effect was not obvious during the storage. The sensory value of the control sausage was decreased by $42.12 \%$ when stored for 30 days, while sausages added with BTE was decreased by only about $13.34 \%$. BTE showed more potential in Cantonese sausage preservation and the sausage with more than $0.15 \%$ addition of BTE had twice shelf-life $\left(40^{\circ} \mathrm{C}\right)$ more than that of $0.02 \%$ sodium nitrite sausage and five times more than that of control group. Adding BTE in sausage could be a good potential for reducing the usage of nitrite in meat products.
\end{abstract}

Keywords: Black Tea Extract (BTE), Cantonese Sausage, Preservation

\section{Introduction}

Nitrite is an important food additive in the processing of meat products due to its excellent antibacterial and antioxidant effects (Majou and Christieans, 2018; Berardo et al., 2016). It can prevent the lipid oxidation of sausage. However, excessive consumption of nitrite will cause adversary health effects such as carcinogenesis, teratogenesis and mutagenicity. It is necessary to search for a natural extract to replace nitrite partly or completely in sausage for food safety consideration.

Black Tea Extract (BTE), a natural tea extract, has various bioactive characteristics such as anti-cancer and antioxidant properties (Kopjar et al., 2015; Zhao and Shah, 2016; Zhu et al., 2017; Jongberg et al., 2013). A lot of reports have described its potential applications in sausage. It is an excellent choice to replace 2-(1,1-
Dimethylethyl)-1,4-benzenediol (TBHQ), 2,6-di-tertbutyl-4-methylphenol (BHT) and nitrite due to its antioxidant properties and as a natural pigment to protect color (Jayawardana et al., 2019; Lorenzo et al., 2014). Jayawardana et al. (2019) reported that adding black tea extract 0.05 and $0.30 \%$ could reduce the TBARS value in uncured pork sausages without compromising the sensory quality. Meanwhile, the addition of BTE to sausages by replacing the chemically synthetic antioxidants and colorants showed a higher product quality and safety (Brewer, 2011; Estévez, 2011; Shah et al., 2014).

Tea polyphenols may be the main antioxidant components of the Black Tea Extract (BTE), which was related to antioxidant, antimicrobial activity, anti-cancer etc.. The proportion of different components in tea polyphenols may affect its biological activity. There are 
few report about BTE effect on sausage with its tea polyphenols detailed components.

In this study, the total tea polyphenols in BTE was detected by spectrophotometry, the tea catechin in BTE was analyzed by HPLC. Cantonese sausages of five treatments with different BTE dosages were prepared. The acid value, peroxide value and sensory quality of the tea sausages were determined, compared to the control, the sausage with the addition of sodium Nitrite (NIT) and the addition of NIT and BTE. The shelf-life of the sausage was predicted and the preservation effect of BTE on Cantonese sausage was evaluated.

\section{Materials and Methods}

\section{Experimental Design}

The BTE was purchased from Guangdong Haofa Biological Technology Co. Ltd. (Guangzhou, China). Catechin (C), Epicatechin (EC), Caffeine (CAFF), Catechin Gallate (CG), Gallic Acid (GA), Epicatechin Gallate (ECG), Gallocatechin (GC), Epigallocatechin (EGC), Gallocatechin Gallate (GCG), Epigallocatechin Gallate (EGCG) were obtained from Sigma (USA). The tea polyphenols in BTE was determined according to the national standard GB8313 (China National Standardization Committee, 2018). The tea catechin in BTE was analyzed by HPLC (Shimadzu Corporation, Kyoto, Japan) and the conditions were at $280 \mathrm{~nm}$ by Shimadzu SPD ultraviolet detector, C18 column (5 $\mu \mathrm{m}, 4.6 \times 250 \mathrm{~mm}$; Agilent Technologies, Inc., Santa Clara, CA, USA), injection volume $10 \mu \mathrm{L}$, column temperature $20^{\circ} \mathrm{C}$.

Preparation of Cantonese sausage: The raw materials of sausage are from the local market. $60.61 \%$ port lean and $25.97 \%$ fat were cut into a strip about $1 \mathrm{~cm}$ square and mixed with $1.73 \%$ salt, $2.16 \%$ rice wine, $4.33 \%$ soy sauce and $5.19 \%$ sugar. After curing for $30 \mathrm{~min}$, the meat mixtures were stuffed into casings. Raw sausages were tied into knots every $15 \mathrm{~cm}$ and prick $3-5$ pinhole every $1 \mathrm{~cm}$. They were dried at $60^{\circ} \mathrm{C}$ for $48 \mathrm{~h}$ and flipped every $6 \mathrm{~h}$. The mature sausages were stored at $40^{\circ} \mathrm{C}$ for 36 days in an incubator.

Eight treatments for pork sausages: Control (basic formulation); basic formulation with the addition of $0.05, \quad 0.10,0.15, \quad 0.20$ and $0.25 \%$ BTE; basic formulation with the addition of $0.02 \%$ sodium Nitrite (NIT) and basic formulation with the addition of $0.01 \%$ NIT and $0.01 \%$ BTE.

\section{Physicochemical Properties Analysis}

Lipid oxidation and the acid value were measured in triplicate according to the national standard GB5009 (Ministry of Health of China, 2016). Color analysis was carried out in triplicate using a portable spectrophotometer (HunterLab ColorFlex EZ, USA), by measuring a* (red intensity) values of the CIE system.

\section{Prediction of Shelf-Life}

The peroxide value was detected every 6-8 days and the prediction of sausages shelf-life at $40^{\circ} \mathrm{C}$ was studied. The Arrhenius equation was used to the prediction of sausages shelf-life.

\section{Sensory Evaluation}

A panel of 10 assessors aged from 22 to 40 years was trained in descriptive evaluation of sausages. An affective acceptance test was carried out by using a 9point scale (Baldin et al., 2016).

\section{Data Analysis}

Objective measurements were replicated triplicate. The experimental design of lipid oxidation, the acid value, color value and sensory evaluation were by using Complete Randomized Design (CRD). Data Processing System (DPS) software v18.10 (Tang and Zhang, 2013) was applied to analyze the data. Means were compared using Duncan's Multiple Range Test. Differences at $\mathrm{P}<0.05$ were considered significant.

\section{Results and Discussion}

\section{The Tea Polyphenol in BTE}

The polyphenol content of BTE was $26.61 \%$. Gallic acid, caffeine and 8 catechins were identified by HPLC and the results showed that the EGCG content was the highest (Table 1). The order of polyphenol content was: EGCG $>$ EGC $>$ GCG $>$ ECG $>$ GC $>$ EC $>$ C $>$ CG .

The tea polyphenol has showed antioxidant and antimicrobial activity. Wenjiao et al. (2014) reported that it had positive effect on sausage quality. The BTE used in the experiment had more EGCG percentage content than the tea samples reported (Nian et al., 2019). The polyphenol components of different kind of tea is very different (Nian et al., 2019; Wang et al., 2010).

\section{Physicochemical Properties}

The changes of acid value of sausage during the storage are showed in Fig. 1. There was no significant difference among the treatments at first. The acid value of all treatments increased with time. The higher concentration of BTE added, the slower the increase of the acid value. When the concentration of BTE reached $0.15 \%$, statistical analysis showed that the acid value in the sausage was significantly lower compared to the control $(\mathrm{P}<0.05)$. The samples with addition of $0.02 \%$ NIT and the addition of $0.01 \%$ NIT and $0.1 \%$ BTE showed positive effect on the acid value too $(\mathrm{P}<0.05)$. The higher acidity means lower $\mathrm{pH}$. This study was in good agreement those of reported by (Jayawardana et al., 2019; Lorenzo et al., 2014). The change of the acid value might due to the fat and 
protein breakdown by the bacterial activity. These results indicated that nitrite and black tea extract had synergistic inhibition of acid value increase.

The changes of lipid oxidation of sausage during the storage are showed in Fig. 2. The lipid oxidation of all treatments were increased with time and the peroxide value of the control group had the fastest rising rate and exceeded the national limit (show the limit value here) within 36 days, while the sausages with different concentrations of BTE and nitrite were still within the national limit. Different additives have different effects on the initial peroxide values of the sausages. This result conformed to the study by (Jayawardana et al., 2019). The addition of BTE and NIT have inhibitory effect on the lipid peroxide value. The higher concentration of BTE added, the slower the increase of the peroxide value. The ratio of the final to initial peroxide value on the 36th storage day were 29.20 (control), 3.90 (0.05\% BTE), 2.38 (0.10\% BTE), 1.89 (0.15\% BTE), 1.82 (0.20\% BTE), 1.88 (0.25\% BTE), 4.42 (0.02\% NIT), 3.61 $(0.01 \% \mathrm{NIT}+0.10 \% \mathrm{BTE})$ respectively. When the concentration of the BTE was increased to $0.15 \%$, the inhibitory effect of the BTE on the peroxide value tended to be stable. Jongberg et al. (2013; Jayawardana et al., 2019) also observed the green tea and black tea extract could inhibit TBARS values increasing of sausage. Furthermore, (Jayawardana et al., 2019) showed that the sausage of $0.2 \%$ and $0.3 \%$ BTE addition had significantly lower TBARS compared to the $0.1 \%$ BHT sample. In this study, the lipid oxidation inhibitory had no significant different $(\mathrm{P}<0.05)$ between the samples of BTE $(0.20 \%$ or $0.25 \%)$ and NIT $(0.02 \%)$ and between the samples of BTE $(0.20 \%$ or $0.25 \%)$ and NIT $(0.01 \%)$ with BTE $(0.01 \%)$ on the $36^{\text {th }}$ day. The lipid oxidation reduction might be attributed to the polyphenols in BTE (Jukić et al., 2015; Predescu et al., 2018), as detected above.

Figure 3 shows the $a^{*}$ values of the CIE system of sausage. During the storage period, the chroma value first decreased and then increased for all samples. On the 16th day, there were no significant different between the control sample and the $0.02 \%$ NIT sample, but after that, the a* value of $0.02 \%$ NIT samples increased faster than the control samples and there were significant different $(\rho<0.05)$ between them. This may be related to the coloration mechanism of meat products $(\mathrm{Hu}, 2016)$.

\section{Prediction of Sausages Shelf-Life}

According to the Arrhenius empirical formula and based on the lipid peroxidation value, the first-order dynamic equation:

$$
\text { Ln }(\text { Lipid oxidation value })=a \times \text { time }+b
$$

where, $a$ is the reaction rate constant, $b$ is natural logarithm of the initial peroxide value.

By detemining the peroxide value of sausage at different times, the prediction of sausages shelf life at $40^{\circ} \mathrm{C}$ under different treatment conditions can be calculated (Table 2).

The shelf life of the control group was predicted to be 43 days (Table 2), while the sausage with $0.05 \%$ BTE had a shelf life of 117 days, which was longer than the sausages with $0.02 \%$ nitrite (107 days). The shelf life of sausage with more than $0.15 \%$ BTE had more than 240 days of predicted shelf life, which was more than twice to the shelf life of $0.02 \%$ NIT sample and five times the shelf life of the control. Therefore, the Cantonese sausage with the addition of BTE only was an acceptable meat product, in terms of shelf life.

\section{Sensory Evaluation}

Sensory evaluation data of the samples are presented in Fig. 4. The sensory value of the control sausage was decreased by $42.12 \%$ when stored for 30 days, while sausages added with BTE was decreased by about $13.34 \%$ only. Statistical analysis indicated that there is no significant different $(\mathrm{P}<0.05)$ among the sausage of the addition of $0.15,0.20,0.25 \%$ BTE, $0.02 \%$ NIT and $0.01 \%$ NIT with $0.10 \%$ BTE on the 15 th day and among the sausage of the addition of $0.20 \%$ BTE, $0.02 \%$ NIT and $0.01 \%$ NIT with $0.10 \%$ BTE on the 30th day. The sausages added BTE showed less rancidity after 4 weeks' storage compared to the control group. BTE with strong antioxidant and antimicrobial properties might have affected the rate of sausage spoilage processes (Almajano et al., 2008; Chen and Ho, 1995; Friedman, 2007). The concentrations of BTE or NIT have an important impact on sensory acceptance of the Cantonese sausages.

Table 1: Composition and contents of polyphenols from the black tea extract

\begin{tabular}{lc}
\hline The composition & Contents (\%) \\
\hline GA & $0.27 \pm 0.04$ \\
GC & $1.88 \pm 0.27$ \\
EGC & $5.31 \pm 0.70$ \\
CAFF & $5.75 \pm 0.71$ \\
C & $0.87 \pm 0.13$ \\
EC & $1.66 \pm 0.23$ \\
EGCG & $10.23 \pm 1.39$ \\
GCG & $2.69 \pm 0.37$ \\
ECG & $2.54 \pm 0.20$ \\
CG & $0.34 \pm 0.04$ \\
\hline C: Catechin, EC: Epicatechin, CG: Catechin Gallate, EGC: \\
Epicatechin Gallate, GC: Gallocatechin, EGC: \\
Epigallocatechin, GCG: Gallocatechin Gallate, EGCG: \\
Epigallocatechin Gallate; GA: Gallic Acid, CAFF: Caffeine
\end{tabular}


Huan Cheng et al. / American Journal of Biochemistry and Biotechnology 2020, 16 (3): 417.423 DOI: 10.3844/ajbbsp.2020.417.423

Table 2: Prediction of sausages shelf-life at $40^{\circ} \mathrm{C}$

\begin{tabular}{lllll}
\hline Samples & $\mathrm{a}$ & $\mathrm{b}$ & $\mathrm{R}^{2 \mathrm{c}}$ & Shelf-life (day) \\
\hline $0 \%$ BTE & 0.0948 & 3.408 & 0.961 & 43 \\
$0.05 \%$ BTE & 0.0337 & 3.264 & 0.922 & 117 \\
$0.10 \%$ BTE & 0.0224 & 3.091 & 0.980 & 169 \\
$0.15 \%$ BTE & 0.0156 & 3.061 & 0.954 & 240 \\
$0.20 \%$ BTE & 0.0154 & 3.096 & 0.928 & 246 \\
$0.25 \%$ BTE & 0.0156 & 3.131 & 0.907 & 245 \\
$0.02 \%$ NIT & 0.0439 & 4.012 & 0.924 & 107 \\
$0.01 \%$ NIT+0.1\% BTE & 0.0323 & 4.005 & 0.965 & 145 \\
\hline
\end{tabular}

c. Correlation coefficients of determination

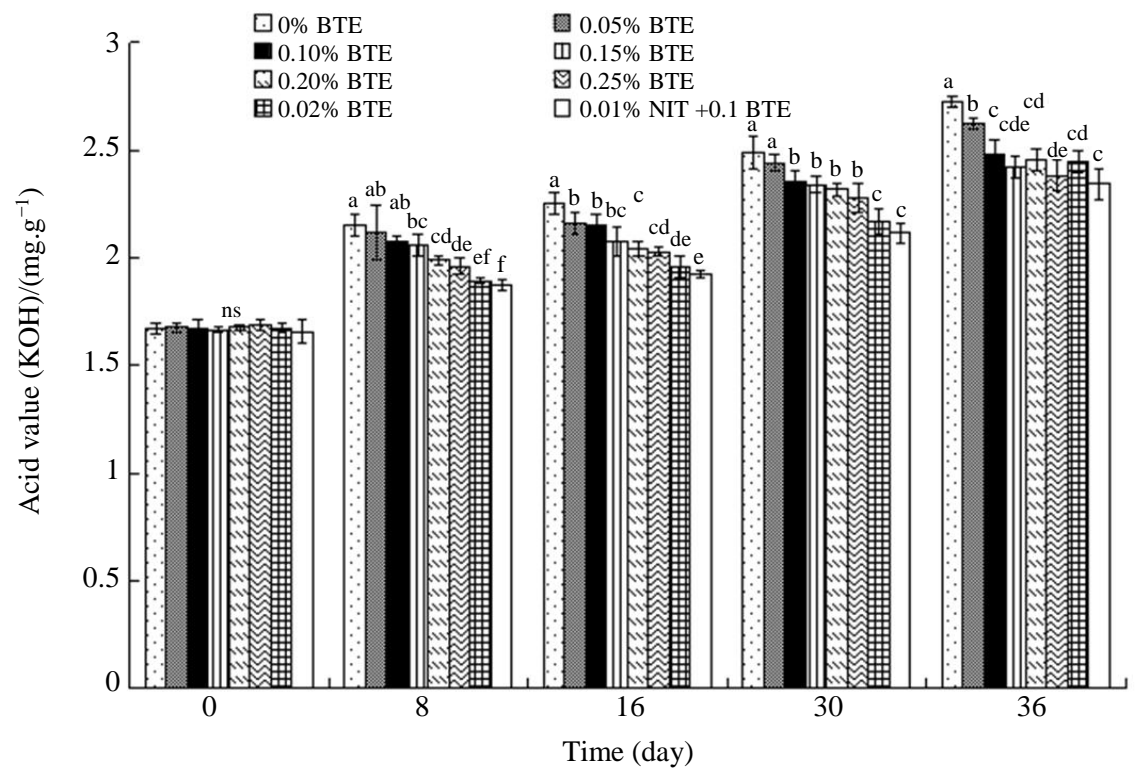

Fig. 1:Effect of BTE on the acid values of Sausage. a-f: Different letters indicate significant differences between the various treatments at the same time. NS indicates no significant differences at the 0.05 level

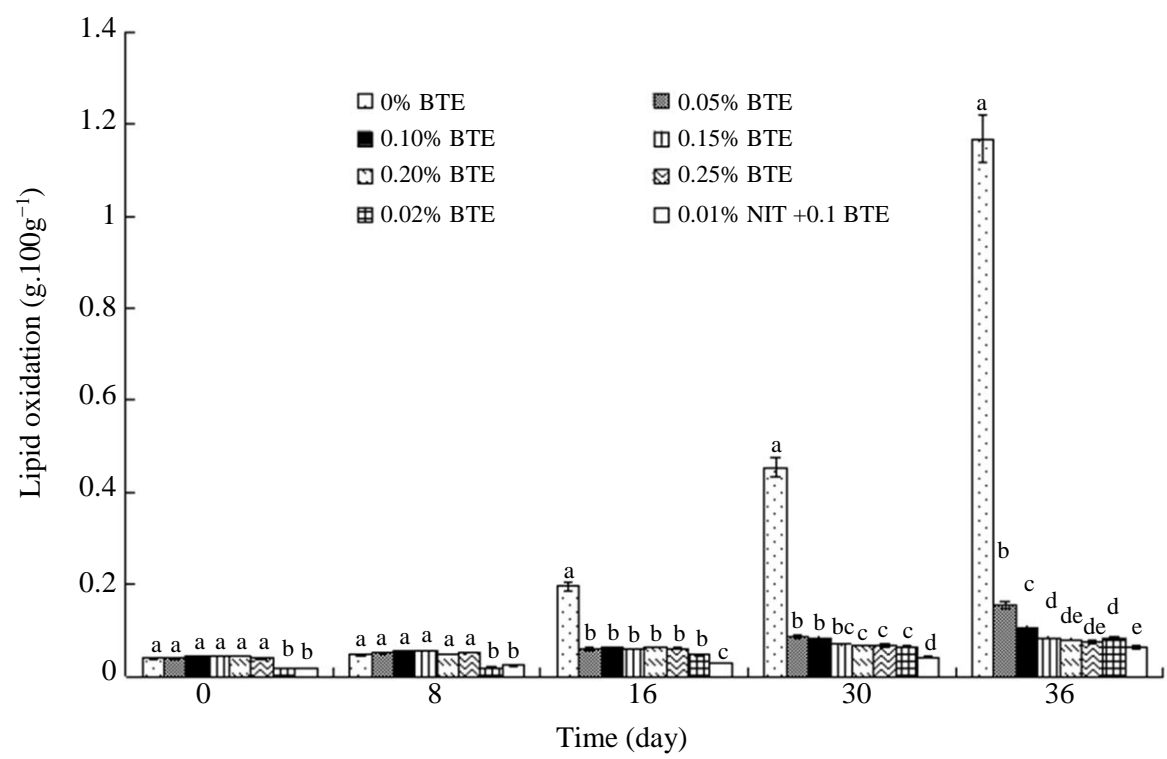

Fig. 2: Effect of BTE on the lipid oxidation of Sausage. a-e: Different letters above bars indicate significant differences between the various treatments at the same time 


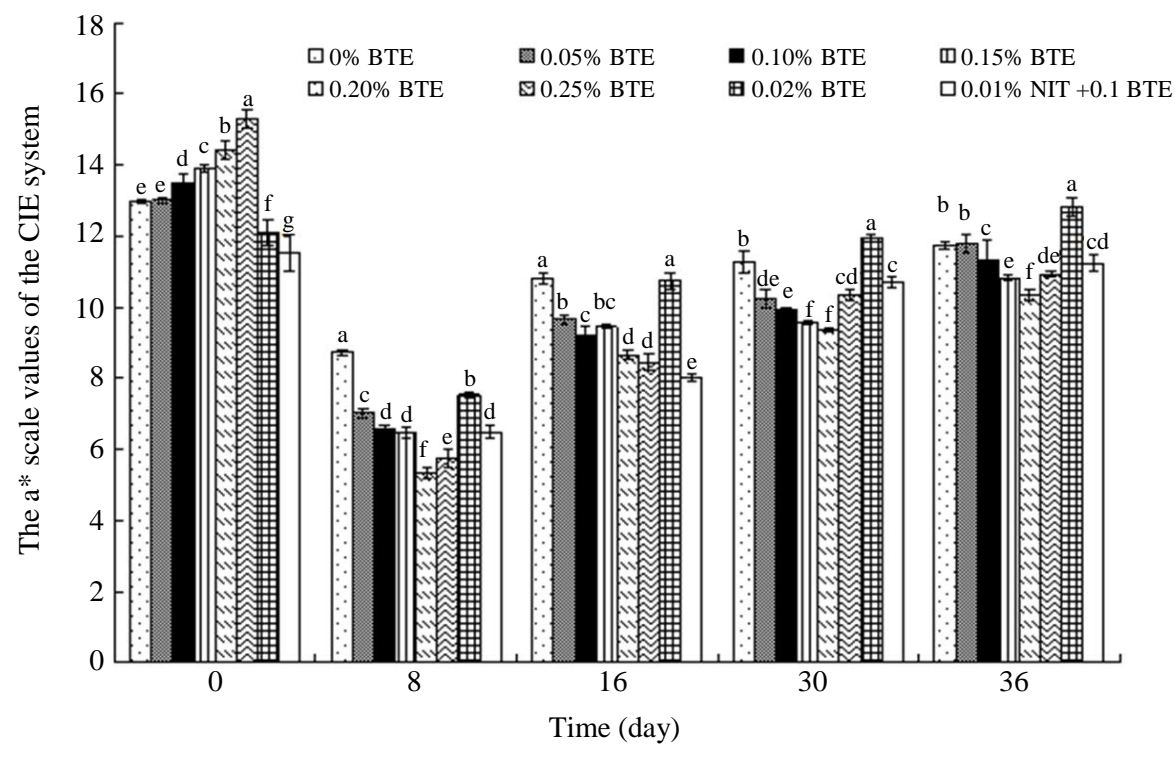

Fig. 3: Effect of BTE on the color of Sausage. a-g: Different letters above bars indicate significant differences between the various treatments at the same time

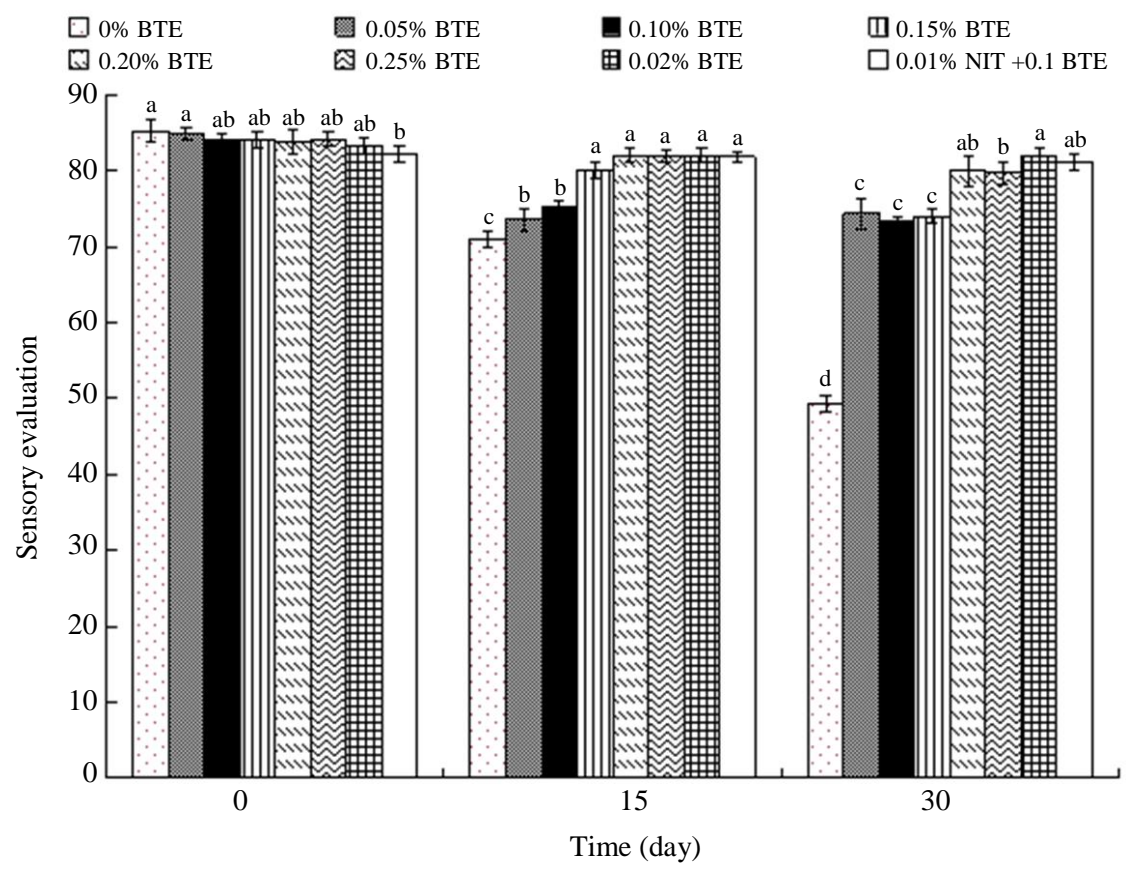

Fig. 4: Effect of BTE on sensory evaluation of Sausage. a-c: Different letters above bars indicate significant differences between the various treatments at the same time

\section{Conclusion}

Black Tea Extract (BTE) was rich in tea polyphenols, which was related to antioxidant, antimicrobial activity, anti-cancer et al. This paper studied the effect of BTE on the preservation of Cantonese Sausage based on polyphenol components analysis. According to the study, the Black Tea Extraction (BTE) had $26.61 \%$ polyphenols. The components were gallic acid $(0.27 \%)$, caffeine $(5.75 \%)$ and 8 catechins, which included Catechin $(0.87 \%)$, epicatechin $(1.66 \%)$, catechin gallate $(0.34 \%)$, epicatechin gallate (2.54\%), gallocatechin (1.88\%), epigallocatechin $(5.31 \%)$, gallocatechin gallate $(2.69 \%)$, epigallocatechin gallate $(10.23 \%)$. Compared with the 
control, the sausage with $0.15 \%$ BTE had significantly improved the product quality by reducing the acid value and lipid oxidation value. The nitrite and BTE had synergistic inhibition of acid value increase. When the BTE concentration increased to $0.15 \%$, the inhibitory effect on the peroxide value tended to be stable. The BTE had the positive effect on the color of the freshly prepared sausage, but this effect was not obvious during the storage. The sausages added BTE showed less rancidity after 4 weeks' storage compared to the control group. The sensory value of the control sausage was decreased by $42.12 \%$ when stored for 30 days, while sausages added with BTE was decreased by about $13.34 \%$ only. Sensory evaluation had no significant different $(\rho<0.05)$ among the sausage of the addition of $0.20,0.25$ BTE, $0.02 \%$ NIT on the 15th and 30th day. The shelf life of sausage with more than $0.15 \%$ BTE had longer predicted shelf life, which was more than twice to that of $0.02 \%$ NIT and five times to that of the control. The development of nitrite free sausage with BTE could be promising in the future processed meat product market.

\section{Acknowledgement}

This research was supported by the national science and technology support program of China (Grant No. 2014BAD04B01) and the Teaching Reform Project of Zhejiang University "Food production design and comprehensive training".

\section{Author's Contributions}

Huan Cheng and Dan Wu: Participated in all experiments, coordinated the data-analysis and contributed to the writing of the manuscript.

Ying Liu, Xiaona Zhao and Chenlan Mao: Participated in all experiments.

Shuping Wang and Jianchu Chen: Grammar checking and modification.

\section{Conflict of Interest}

The authors had no conflict of interest to declare.

\section{References}

Almajano, M. P., Carbo, R., Jiménez, J. A. L., \& Gordon, M. H. (2008). Antioxidant and antimicrobial activities of tea infusions. Food chemistry, 108(1), 55-63.

Baldin, J. C., Michelin, E. C., Polizer, Y. J., Rodrigues, I., de Godoy, S. H. S., Fregonesi, R. P., ... \& Fernandes, A. M. (2016). Microencapsulated jabuticaba (Myrciaria cauliflora) extract added to fresh sausage as natural dye with antioxidant and antimicrobial activity. Meat Science, 118, 15-21.
Berardo, A., De Maere, H., Stavropoulou, D. A., Rysman, T., Leroy, F., \& De Smet, S. (2016). Effect of sodium ascorbate and sodium nitrite on protein and lipid oxidation in dry fermented sausages. Meat science, 121, 359-364.

Brewer, M. S. (2011). Natural antioxidants: sources, compounds, mechanisms of action and potential applications. Comprehensive reviews in food science and food safety, 10(4), 221-247.

Chen, C. W., \& Ho, C. T. (1995). Antioxidant properties of polyphenols extracted from green and black teas. Journal of food lipids, 2(1), 35-46.

China National Standardization Committee, 2018. Determination of total polyphenols and catechins content in tea. Standardization Administration of China (GB/T8313-2018). China National Standard, Beijing, China. https://www.chinesestandard.net/PDF/English.aspx/ GBT8313-2018

Estévez, M. (2011). Protein carbonyls in meat systems: A review. Meat science, 89(3), 259-279.

Friedman, M. (2007). Overview of antibacterial, antitoxin, antiviral and antifungal activities of tea flavonoids and teas. Molecular nutrition \& food research, 51(1), 116-134.

$\mathrm{Hu}, \mathrm{H}$. (2016). Progress in coloration mechanism and color assessment of meat products. Meat Research (in Chinese), 30, 48-53.

Jayawardana, B. C., Warnasooriya, V. B., Thotawattage, G. H., Dharmasena, V. A. K. I., \& Liyanage, R. (2019). Black and green tea (Camellia sinensis L.) extracts as natural antioxidants in uncured pork sausages. Journal of Food Processing and Preservation, 43(2), e13870.

Jongberg, S., Tørngren, M. A., Gunvig, A., Skibsted, L. H., \& Lund, M. N. (2013). Effect of green tea or rosemary extract on protein oxidation in Bologna type sausages prepared from oxidatively stressed pork. Meat science, 93(3), 538-546.

Jukić, H., Hrnjica, D., \& Aldžić, A. (2015). THE INFLUENCE AND THE ROLE OF POLYPHENOLS ON THE SENSORY AND ORGANOLEPTIC ASPECTS OF FOOD. Hrana $u$ zdravlju i bolesti: znanstveno-stručni časopis za nutricionizam i dijetetiku, 4(2), 113-119.

Kopjar, M., Tadić, M., \& Piližota, V. (2015). Phenol content and antioxidant activity of green, yellow and black tea leaves. Chemical and Biological Technologies in Agriculture, 2(1), 1.

Lorenzo, J. M., Sineiro, J., Amado, I. R., \& Franco, D. (2014). Influence of natural extracts on the shelf life of modified atmosphere-packaged pork patties. Meat Science, 96(1), 526-534.

Majou, D., \& Christieans, S. (2018). Mechanisms of the bactericidal effects of nitrate and nitrite in cured meats. Meat science, 145, 273-284. 
Ministry of Health of China, 2016. National Food Safety Standard. Standardization Administration of China (GB/T 5009-2016). China National Standard, Beijing,

China. https://www.mpi.govt.nz/dmsdocument/14395/send

Nian, B., Chen, L., Yi, C., Shi, X., Jiang, B., Jiao, W., ... \& Zhao, M. (2019). A high performance liquid chromatography method for simultaneous detection of 20 bioactive components in tea extracts. Electrophoresis, 40(21), 2837-2844.

Predescu, C., Papuc, C., CarmenPETCU, G. G., \& Rus, A. E. (2018). The Effect of Some Polyphenols on Minced Pork during Refrigeration Compared with Ascorbic Acid. Bulletin UASVM Food Science and Technology, 75, 1.

Shah, M. A., Bosco, S. J. D., \& Mir, S. A. (2014). Plant extracts as natural antioxidants in meat and meat products. Meat science, 98(1), 21-33.

Tang, Q. Y., \& Zhang, C. X. (2013). Data Processing System (DPS) software with experimental design, statistical analysis and data mining developed for use in entomological research. Insect Science, 20(2), 254-260.
Wang, Y., Yang, X., Li, K., Li, C., Li, L., Li, J., ... \& Song, X. (2010). Simultaneous determination of theanine, gallic acid, purine alkaloids, catechins and theaflavins in black tea using HPLC. International journal of food science \& technology, 45(6), 1263-1269.

Wenjiao, F., Yunchuan, C., Junxiu, S., \& Yongkui, Z. (2014). Effects of tea polyphenol on quality and shelf life of pork sausages. Journal of Food science and Technology, 51(1), 191-195.

Zhao, D., \& Shah, N. P. (2016). Synergistic application of black tea extracts and lactic acid bacteria in protecting human colonocytes against oxidative damage. Journal of agricultural and food chemistry, 64(11), 2238-2246.

Zhu, C., Tai, L. L., Wan, X. C., Li, D. X., Zhao, Y. Q., $\& \mathrm{Xu}, \mathrm{Y}$. (2017). Comparative effects of green and black tea extracts on lowering serum uric acid in hyperuricemic mice. Pharmaceutical biology, 55(1), 2123-2128. 